\title{
Participación social rural en la mitigación de la vulnerabilidad derivada de la variabilidad climática en Colombia ${ }^{1}$
}

Yanine Rozo Leguizamón ${ }^{2}$

Corpoica

\section{Artículo científico}

Material original autorizado para su primera publicación en el Journal de Ciencias Sociales, Revista Académica de la Facultad de Ciencias Sociales de la Universidad de Palermo.

\section{Recibido: 23-3-2016}

Aceptado: 18-10-2016

Resumen: El nivel de vulnerabilidad está relacionado con el tipo de riesgo, el grado de amenaza y el nivel socioeconómico de la población afectada. Depende, por tanto, de la posibilidad que tienen los individuos o las comunidades de sobreponerse a la situación y del reconocimiento de la importancia que para ellos representan las posibles amenazas y las diferentes formas de afrontarlas. El presente artículo presenta los resultados del proceso investigativo desarrollado en el Altiplano Cundiboyacense de Colombia, con la pretensión de identificar la percepción que tienen los campesinos productores de leche sobre las afectaciones derivadas de la variabilidad climática en su sistema de producción, y los significados que le otorgan a "vulnerabilidad", "riesgo" y "amenaza". Se parte de su reconocimiento como sujetos y actores sociales protagonistas importantes del desarrollo de la comunidad rural en la que viven y conviven en el día a día.

El estudio se realizó con la participación de los miembros de un equipo de investigadores de la Corporación Colombiana de Investigación Agropecuaria - Corpoica bajo la dirección de la Ph.D. Elizabeth Aguilera Garramuño, con la participación de la Magister y autora de este artículo Yanine Rozo Leguizamón, del Magister Edwin Oswaldo Rojas y de la Profesional de Apoyo a la Investigación Estefanía Fajardo Rojas.

Palabras clave: Variabilidad Climática, vulnerabilidad, riesgo, amenaza, resiliencia.

\footnotetext{
1 Este material es una ampliación y profundización de la ponencia titulada "Variabilidad climática en Colombia y participación activa y efectiva del campesinado en la prevención de la vulnerabilidad derivada" presentada en el IX Congreso Sociedades Rurales Latinoamericanas: Diversidades, Contrastes y Alternativas, de la Asociación Latinoamericana de Sociología Rural - ALASRU 2014. Autorizada para su primera publicación en Journal de Ciencias Sociales Revista Académica de la Facultad de Ciencias Sociales de la Universidad de Palermo.

2 Ingeniera Agrónomo de la Universidad de Cundinamarca de Colombia. Magíster en Planeación Socioeconómica de la Universidad Santo Tomás de Colombia. Investigador Máster de la Corporación Colombiana de Investigación Agropecuaria - Centro de Investigación La Suiza. Sede Cúcuta. Correo electrónico: yrozo@corpoica.org.co
} 
Abstract: The level of vulnerability is related to the type of risk, the degree of threat and socioeconomic status of the affected population. It depends, therefore, the possibility that individuals or communities to overcome the situation and recognition of the importance they represent potential threats and different ways of addressing them. This article presents the results of the research process developed in the Altiplano Cundiboyacense of Colombia, with the aim of identifying the perception of the peasant producers of milk on the damages resulting from climate variability in the production system, and the meanings that awarded to "vulnerability", "risk" and "threat". It is part of their recognition as subjects and social actors important players in the development of the rural community in which they live and coexist in the day.

The study was realized with the participation of members of a team of researchers from the Colombian Corporation for Agricultural Research - Corpoica under the direction of the Ph.D. Elizabeth Aguilera Garramuño, with the participation of the Magister and author of this article Yanine Rozo Leguizamón, Magister Edwin Oswaldo Rojas and Professional Support Research Estefania Rojas Fajardo.

Key words: Climate variability, vulnerability, risk, threat, resilience. 


\section{Introducción}

El Altiplano Cundiboyacense colombiano es afectado por la llamada "Ola Invernal”, evento climático de lluvias extremas asociado con el fenómeno La Niña, que generó lluvias hasta 38\% superiores al promedio nacional histórico, entre junio de 2010 y abril de 2012, provocando inundaciones en el departamento de Boyacá en aproximadamente 13,490 ha (3,3\% del área total del departamento) y en Cundinamarca en 5,584 ha inundadas, (5,3\% del área del total de departamento), áreas dedicadas en un 77\% a pastos para el ganado de leche (Corpoica 2012b). Esta situación conlleva al gobierno nacional, por medio del Decreto 4580 de 2010, a declarar un desastre nacional y establecer el Estado de Emergencia Económica, Social y Ecológica, con el fin de enfrentar esta calamidad pública.

La investigación en cuyos resultados se fundamenta este artículo se realiza con el propósito de comprender los significados que los campesinos productores de leche del Altiplano Cundiboyacense de Colombia otorgan a sus prácticas culturales para enfrentar las "amenazas" asociadas con la variabilidad climática y disminuir la "vulnerabilidad" de sus sistemas productivos, con el fin de reducir el riesgo que estas amenazas representan para el ejercicio de sus actividades productivas. Este trabajo se adelanta en el marco de un macro proyecto financiado por Colciencias titulado "Zonificación Agroclimática del Sistema de Ganadería del Valle de Ubaté y Chiquinquirá y la región del Alto Chicamocha", cuyos resultados pretenden apoyar la planificación de actividades productivas, de investigación y capacitación, de acuerdo con los impactos de la variabilidad climática.

Los métodos y técnicas de análisis utilizados -observación participante, encuestas cerradas, talleres de vulnerabilidad y entrevistas semiestructuradas-, se asumen como un referente metodológico importante. En este sentido, se pretende contribuir a la reducción de la vulnerabilidad de los sectores productivos campesinos derivada del desconocimiento de la forma de interpretar acertada y oportunamente las amenazas y riesgos asociados con la variabilidad climática. 


\section{Fundamentación teórica y normativa}

\section{Del sujeto al Actor Social}

Inicialmente se parte de la tesis de "sujeto-actor social" de Alain Touraine $(1996,2000)$ con la que se pretende validar la participación activa y efectiva de los productores de leche en el contexto de objeto de estudio, es decir, su reconocimiento como sujetos y actores sociales válidos para ser consultados sobre sus percepciones del fenómeno natural, sus impactos y acciones para su mitigación. En efecto, el interrogante sobre la percepción que los habitantes de la zona rural andina, en este caso los productores de leche del Altiplano Cundiboyacense, tienen respecto del fenómeno de variabilidad climática y de vulnerabilidad, y la amenaza que representa su presencia cada vez más constante para sus medios y calidad de vida, la de sus familias y comunidad en general, hace necesario acudir a estas tesis en las que el Sujeto se asume como combinación de una identidad personal y una cultura particular, con la participación en un mundo racionalizado, y como afirmación de su libertad y su responsabilidad (Touraine, 1996, 2000).

Reconocerles como sujetos es reconocerles como protagonistas del desarrollo de su propia vida, es identificarles como personas capaces de tener proyectos, de elegir, de juzgar de modo positivo o negativo, y también de tener relaciones sociales "ya se trate de relaciones de cooperación, de consenso o conflictivas" (Touraine, 1996, p. 41; 2000, pp. 65-66). Estas capacidades les facilita el camino hacia su configuración como actores sociales, pero no su logro, dado que para ello deben adquirir "conciencia ciudadana", la que se adquiere cuando perciben que su participación y opiniones son tenidas en cuenta por quienes tienen la autoridad para tomar decisiones en un momento determinado y perciben, también, que esas decisiones se implementan y evalúan con su activa y efectiva participación en las decisiones que, en ese sentido, afectan o pueden afectar sus vidas y las de sus familias y colectividad.

En esta investigación se reconoce, entonces, a los productores rurales, específicamente a los productores de leche del Altiplano Cundiboyacense como sujetos o protagonistas en el desarrollo de su propia vida, es decir, como personas capaces de tener proyectos productivos (producción de leche) y de elegirlos y juzgarlos de modo positivo o negativo según su conveniencia para el desarrollo óptimo de las actividades que realizan cotidianamente. Son por tanto personas válidas para expresar sus percepciones sobre la variabilidad climática, sus efectos en la producción y en la comunidad, y sobre lo que consideran necesario y útil para la mitigación y control de los impactos derivados. Su capacidad surge de su propia experiencia, de sus interacciones con la comunidad o el entorno social en el que se encuentran insertos y de su diario vivir, lo que le permite plantear sus propias opiniones, sensaciones, recuerdos, sentimientos a partir de su percepción particular y propia. 
Se les reconoce también como actores sociales o protagonistas del desarrollo de la sociedad rural en la que se encuentran insertos, cuando además de consultarles sobre esas percepciones, ellas son tenidas en cuenta por quien tiene o ejerce el poder de tomar decisiones en un momento determinado impulsando la configuración en ellos de conciencia ciudadana. En este sentido, la investigación pretende no sólo comprender el sentido o el significado que el productor lechero en el contexto del estudio les otorga a los fenómenos citados, sino tenerlos en cuenta para formular participativamente posibles acciones que contribuyan a su mitigación y control.

\section{Vulnerabilidad, Riesgo y Amenaza}

Las categorías de "Vulnerabilidad", "Riesgo", "Amenaza" se abordan inicialmente desde la teoría formal y fundamentos normativos, posteriormente se comparan con los sentidos o significados que los productores de leche les asignan en el entorno rural objeto de estudio. En este sentido, a continuación, se describen los conceptos pertinentes.

En general hay coincidencia en la mayoría de los autores en relación con la definición general de "vulnerabilidad", razón por la cual se acoge el concepto que posiblemente encierra lo que todos ellos pretenden significar: por vulnerabilidad se entiende el proceso multidimensional que confluye en el riesgo o la probabilidad del individuo, hogar o comunidad, de ser herido, lesionado o dañado ante cambios o permanencias de situaciones externas o internas, cambios que no solo afectan al individuo sino a todo su entorno (Busso, 2001, p. 8). Este concepto general, se traduce en Colombia en lo que podría presentarse como el concepto oficial de vulnerabilidad ${ }^{3}$ para el país, dada su presentación a través de la Ley 1523 de 2012, en la que se adopta la Política Nacional de Gestión del Riesgo de Desastres y se establece el Sistema Nacional de Gestión del Riesgo de Desastres. Este artículo define vulnerabilidad de la siguiente forma:

Vulnerabilidad: Susceptibilidad o fragilidad física, económica, social, ambiental o institucional que tiene una comunidad de ser afectada o de sufrir efectos adversos en caso de que un evento físico peligroso se presente. Corresponde a la predisposición a sufrir pérdidas o daños de los seres humanos y sus medios de subsistencia, así como de sus sistemas físicos, sociales, económicos y de apoyo que pueden ser afectados por eventos físicos peligrosos.

Tal y como se aprecia, el nivel de vulnerabilidad está relacionado con el tipo de riesgo y la capacidad de respuesta a una amenaza física, económica, social, ambiental o institucional de una población en un momento determinado, es decir, depende tanto de la posibilidad que tienen los individuos o las comunidades de sobreponerse a la situación como del reconocimiento de la importancia que para ellos representa las posibles amenazas y las formas

\footnotetext{
${ }^{3}$ REPÚBLICA DE COLOMBIA. Congreso de la República (2012). Numeral 27 del artículo 4 de la Ley 1523 del 24 de abril de 2012 , Por la cual se adopta la Política Nacional de Gestión del Riesgo de Desastres y se establece el Sistema Nacional de Gestión del Riesgo de Desastres y se dictan otras disposiciones. URL: http://www.alcaldiabogota.gov.co/sisjur/normas/Norma1.jsp?i=47141
} 
de afrontarlas. Para el caso rural que ocupa la investigación, "vulnerabilidad" se asume entonces como la reducción o eliminación de la habilidad de la población rural de responder acertada y oportunamente, en el sentido de resistir o recobrarse en el menor tiempo posible de las amenazas o presiones climáticas sobre sus medios de producción agrarios y, por tanto, sobre su bienestar, el de sus familias y comunidad rural.

El concepto de "riesgo", por su parte, ha sido asumido por el Instituto de Hidrología, Meteorología y Estudios Ambientales de Colombia (IDEAM), como la probabilidad del incidente y la magnitud de las consecuencias adversas de un evento climático de peligro (IDEAM, 2011). Se expresa como la interacción entre Amenaza y Vulnerabilidad, entendiendo por "Amenaza" el peligro latente asociado a un evento climático en un sitio específico y en un tiempo determinado, y que produzca efectos adversos en personas o en el medio ambiente. También lo expresa como la probabilidad de exceder un nivel de ocurrencia de un evento con cierta intensidad en un determinado sitio y período (IDEAM, 2011).

Estos conceptos se encuentran oficialmente acogidos para Colombia a través de la citada Ley 1523 del 24 de abril de 2012, la cual expresa en su numeral 25 del artículo 4 la definición de riesgo:

Riesgo de desastres: Corresponde a los daños o pérdidas potenciales que pueden presentarse debido a los eventos físicos peligrosos de origen natural, socio-natural tecnológico, biosanitario o humano no intencional, en un período de tiempo específico y que son determinados por la vulnerabilidad de los elementos expuestos; por consiguiente, el riesgo de desastres se deriva de la combinación de la amenaza y la vulnerabilidad.

La misma Ley establece el concepto de amenaza en su numeral 3 del artículo 4, el cual literalmente expresa:

Amenaza: Peligro latente de que un evento físico de origen natural, o causado, o inducido por la acción humana de manera accidental, se presente con una severidad suficiente para causar pérdida de vidas, lesiones u otros impactos en la salud, así como también daños y pérdidas en los bienes, la infraestructura, los medios de sustento, la prestación de servicios y los recursos ambientales.

La variabilidad climática ha causado en Colombia, América Latina y el mundo en general efectos perversos en todo sentido, fundamentalmente en el sector rural en donde los sistemas productivos se han visto afectados, lo cual ha traído consecuencias sociales muy difíciles de superar. No obstante, los habitantes rurales han mostrado una capacidad de adaptación y respuesta que particularmente en Colombia son dignas de resaltar. 


\section{Capacidad de adaptación o resiliencia}

Por "Capacidad de Adaptación" o "Resiliencia" se asume la habilidad de una población para reconocer y responder a los eventos climáticos presentes a través de la reorganización de actividades, inversiones, reubicación de Ios recursos, entre otros aspectos, para minimizar su vulnerabilidad (IDEAM, 2011). Se hace necesario, de acuerdo con este concepto, que el proceso de adaptación esté basado en la percepción de la vulnerabilidad sociocultural, así como en las capacidades locales, las buenas prácticas locales y las alternativas de solución que fortalezcan la resiliencia de las comunidades y sus sistemas de producción.

Adaptación: Comprende el ajuste de los sistemas naturales o humanos a los estímulos climáticos actuales o esperados o a sus efectos, con el fin de moderar perjuicios o explotar oportunidades beneficiosas. En el caso de los eventos hidrometeorológicos, la Adaptación al Cambio Climático corresponde a la gestión del riesgo de desastres en la medida en que está encaminada a la reducción de la vulnerabilidad o al mejoramiento de la resiliencia en respuesta a los cambios observados o esperados del clima y su variabilidad (IDEAM, 2011).

En el caso de esta investigación, de cuyos resultados se deriva este artículo, los cambios agroclimáticos generan distintas formas de percepción y de expresión en los individuos y poblaciones de las áreas afectadas por los riesgos o amenazas climáticas, respecto del deterioro tanto de sus medios de vida como de su calidad de vida. Se trata entonces de identificar esas percepciones a través de la interpretación de lo que, para ellos, como actores sociales válidos para ser consultados, representa el fenómeno natural y los riegos y amenazas que de su presencia se derivan. En este orden, "percepción" se asume en este estudio como la capacidad que tiene el individuo para conocer y comprender el entorno que lo rodea y de actuar dándole sentido a lo que hace.

\section{Proceso metodológico}

Momentos de la investigación. El estudio se realiza en cuatro momentos durante los cuales se desarrollan las actividades pertinentes:

- Primer Momento: Caracterización de los sistemas de producción. En este se realiza un primer acercamiento, desde la teoría formal, a las posibles categorías de análisis del estudio. Igualmente se adelanta un primer acercamiento a la realidad objeto de estudio durante el cual se realizan procesos de observación que van de no participante a participante y se diseñan y aplican encuestas que permitan construir una caracterización del sistema productivo. En este momento se confronta la información obtenida con la teoría formal revisada y se identifican las posibles categorías que orientan las entrevistas, talleres, según el caso. 
Es de aclarar que cuando se habla sobre las "perspectivas de productores" se alude a los siguientes aspectos: experiencias e incidencia histórica de los eventos climáticos extremos; vulnerabilidad de los diferentes componentes del sistema productivo asociada a la variabilidad climática; indicadores de amenaza y riesgos agroclimáticos utilizados por los productores y campesinos, y estrategias tradicionales de manejo y prevención frente a diversos eventos climáticos en el sistema productivo.

- Segundo Momento: el sentido de eventos adversos y sus posibles soluciones. Este constituye la introducción de los investigadores a la realidad objeto de estudio con el fin de identificar las percepciones que sobre las categorías de "Vulnerabilidad" y sobre las subcategorías de "Riesgo", "Amenazas" y "Mecanismos de adaptación y respuesta", tienen los actores sociales sujetos de estudio. Para ello se aplican entrevistas semiestructuradas a una muestra significativa de productores conformadas por 10 personas consideradas “informantes clave" para brindar la información requerida. Sus respuestas son objeto de análisis a través del software Atlas.ti, herramienta de análisis cualitativo que permite trabajar con grandes volúmenes de texto, imágenes, audio y video, y construir mapas relacionales.

- Tercer Momento: Análisis de la Información obtenida con el Atlas.ti. En este se identifican posibles categorías y subcategorías que emergen durante el proceso investigativo con el aporte significativo de los entrevistados y del equipo investigador. Se realiza un proceso de reflexión y construcción en el que se confrontan categorías desde la teoría formal y categorías y subcategorías emergentes con el fin de crear, en últimas, nuevas categorías que permitan abordar distintas alternativas de estrategias para la mitigación y control de eventos adversos en la zona de estudio. Alternativas que se construyen con la activa y efectiva participación de los productores agremiados en sus distintas asociaciones.

La "triangulación" utilizada hace referencia al uso de varios métodos (cuantitativos y cualitativos) y varias fuentes de datos o de enfoques teóricos, que permiten la comparación de la información obtenida y la búsqueda de puntos convergentes, por ejemplo, la triangulación de fuentes de información consiste en comprobar inferencias de una fuente de información a través de otra; para este caso se adelanta la triangulación de instrumentos y técnicas.

\section{Contexto}

El estudio se adelanta en el Valle de Ubaté y Chiquinquirá, su altitud varía entre los 2500 y 3000 m.s.n.m., con una pluviosidad alrededor de los $1000 \mathrm{~mm}$ al año. La microrregión Valle de Ubaté y Chiquinquirá presenta en general tierras de montaña con climas frio y muy frio (Corpoica, 2004). Tiene una extensión de 220.300 ha y comprende 12 municipios del Departamento de Cundinamarca y 5 del Departamento de Boyacá, región que se ha convertido en el 
referente de comercialización de leche cruda en el país (Corpoica, 2012a); y la Región del Alto Chicamocha integrada por 28 municipios de Boyacá, caracterizada por altitudes de 2000 y 3000 msnm, con precipitaciones anuales entre 500 y 1000 mm, zona con excelentes condiciones para la producción de leche (Corpoica, 2012b).
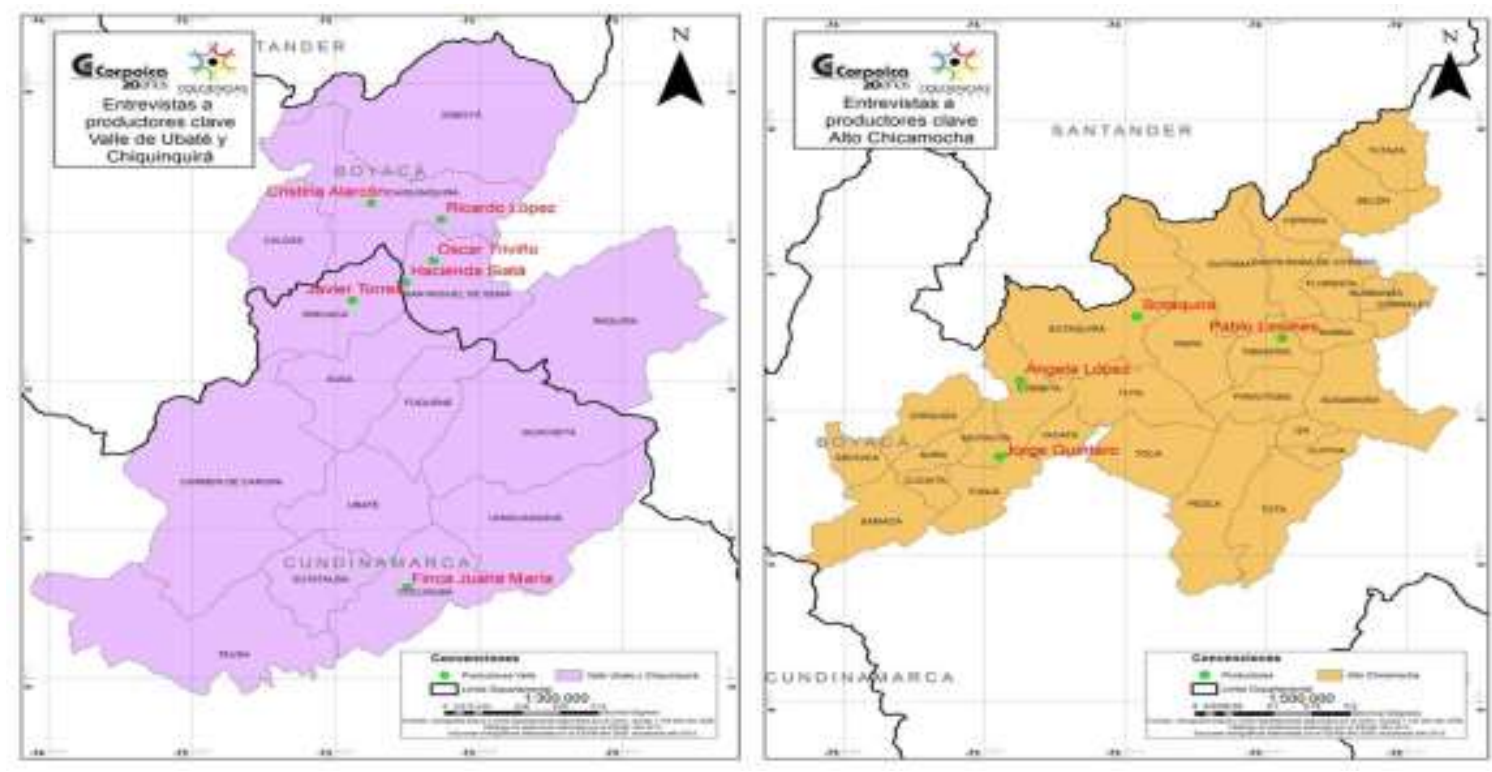

Figura 1. Delimitación de la zona de estudio; Microrregión del Alto Chicamocha (izquierda), Valle de Ubaté y Chiquinquirá (derecha). Fuente: Cartografía Básica 1:100.000 (IGAC, 2006).

La información obtenida en la encuesta permite la construcción de una categorización básica o línea base, que hace posible el establecimiento de una tipología de productores según el área destinada a sus explotaciones lecheras. Se clasifican en pequeños productores los que utilizan de 1 a 5 ha, medianos productores, de 6 a 20 ha, y Grandes productores, de 21 a 50 ha o más. En el Altiplano Cundiboyacense predominan los medianos productores de leche en razón de que cerca del $62 \%$ de los encuestados utilizan entre 6 y 20 ha para sus actividades productivas lecheras. Se puede entonces afirmar que son muy pocos los grandes productores de leche en esta región pues sólo alcanzan el $14,9 \%$ de los encuestados.

La encuesta permite igualmente establecer los eventos climáticos extremos que más afectan a los productores de leche, identificando las heladas y sequías como los de mayor afectación, identificados por más del $50 \%$ de los encuestados, siendo las sequías las que afectan totalmente el área productiva pues las heladas causan una afectación parcial.

Respecto de la intensidad del evento climático adverso se pudo establecer que son los encharcamientos y las inundaciones los eventos de mayor duración en el tiempo pues cerca del $55 \%$ de los encuestados afirma que 
permanecen de 15 días hasta tres meses. Solo el 10,34\% afirma haber permanecido cerca de dos años inundados, principalmente en explotaciones de Chiquinquirá y Ubaté.

En relación con la frecuencia de los eventos climáticos adversos, las heladas son las que ocasionan mayor afectación a las explotaciones en razón de que cerca del $70 \%$ de los encuestados la identifica como el evento más repetitivo al presentarse todos los años. Esta se presenta, de acuerdo con el mayor porcentaje de respuesta, con más alta intensidad principalmente durante los meses de enero $(82,09 \%)$, febrero $(52,50 \%)$ y diciembre $(46,88 \%)$.

De otra parte, la encuesta permite establecer las principales fuentes de información climática consultadas por los productores: el IDEAM, la televisión y la radio, lo mismo que la Red de Información y Comunicación Estratégica del Sector Agropecuario - AGRONET, que tiene como fuente el informe diario del IDEAM; igualmente, algunos buscan información por internet. El 34,88\% afirma que esta información la recibe semanalmente, el 30,23\% que la recibe a diario, el 9,30\% cada 15 días y 2,33\% que la reciben anualmente. El 64,44\% de los productores Ilevan registros del clima, especialmente de precipitaciones, lo que constituye una fortaleza que reafirma su condición de "sujetos" o personas altamente capacitadas para ser consultados sobre estos aspectos.

El $74,47 \%$ de los productores encuestados pertenecen al sector netamente ganadero de producción de leche, el $21,28 \%$ a producción mixta, ganadería y agricultura, y el 4,26\% restante se dedica sólo a la agricultura. El 56,82\% de los productores afirma que producen de 10 a 20 litros vaca/día, el 27,27\% que producen de 5 a 10 litros, el $13,64 \%$ que producen 20 a 40 litros y el 2,27\% más de 60 litros.

\section{Construcción de conocimientos agroclimáticos- percepciones}

\section{Variabilidad Climática}

Los productores ganaderos del Valle de Ubaté y Chiquinquirá, y la Región del Alto Chicamocha consideran importante el conocimiento oportuno de las variaciones en el clima de su región, el cual obtienen por registro de Iluvias que llevan en sus fincas, por afectación a sus sistemas de productivos, o por el diario vivir. Lo plantean así:

Yo creo que hace unos 4 o 5 años más o menos ha empezado como a cambiar, sí, han sido más bruscos los cambios, hay veranos como muy marcados a veces, febreros marcados muy fuertes, $y$ hay inviernos muy fuertes también, pero ya uno no sabe. (P1:RG.1:44) ${ }^{4}$

4 (P1/RG/1:44): es la codificación que se utiliza en adelante, en donde [P1] significa el número del informante; [RG] Iniciales del nombre y apellido del informante; [1:44] número del relato, en este caso, relato 44 del informante 1. 
Desde luego, desde que yo me conozco porque yo soy oriundo de esta región, año tras año se ha visto que el clima ha cambiado, las lluvias son menores, las precipitaciones cada año son menores, y nos vamos de extremo a extremo, de veranos fuertes a inviernos también muy fuertes. (P3:JQ.3:4)

Yo creo que así como muy drásticamente o como que se note mucho, yo creo que como desde el 2007 u 8 ha venido cambiando más drásticamente el clima, ya para el 2010 fue cuanto tuvimos las inundaciones, 10, 11 y 12, el año pasado afortunadamente no llovió tanto y no nos inundamos pero desde el 2007 u 8 empezaron las..., a cambiar el clima pues que era muy notorio ¿no?, lo que te decía, el cambio en la estacionalidad de lluvias, estacionalidad de verano y en la intensidad de estas mismas lluvias y la intensidad del verano. (P7:PL.7:42)

Estos cambios en la variabilidad climática han afectado directamente el sistema productivo, ya que, tras las inundaciones, los cambios en los regímenes de lluvias, en las temperaturas y en los períodos secos y el manejo que los productores hacen de sus praderas, conllevan a afectaciones en las fincas de los productores, a los suelos, a las praderas y al ganado. Los productores expresan lo que consideran, de acuerdo con su experiencia, que ocurre o puede ocurrir en sus predios rurales por los cambios en la variabilidad climática que conllevan al agravar los riesgos asignando explicaciones derivadas de su experiencia que en ningún caso excluyen sus posibles responsabilidades frente a la situación:

Cuatro veces se ha inundado en tres años, afortunadamente no se inunda el 100\%, más o menos un $70 \% . . ., 65$ a $70 \%$, pues como vemos la parte alta, esa que está hacia la vía y hasta allá..., no llega el agua, o no ha llegado en ninguna inundación, entonces nos deja un remanente en pradera o donde parar los animales, afortunadamente no es del 100\%. (P7:P L.7:16) [...] tres o cuatro aguaceritos medio intensos, sin necesidad que sean tan fuertes y ya estamos inundados, ya estamos cargados, ¿por qué?, por lo mismo que te decía antes, cuatro inundaciones yo creo que han apretado la tierra, la han sellado, no sea qué profundidad se habrá sellado y no almacena agua, entonces nos queda como superficial y ahí es donde pues nosotros tenemos inundaciones y todo este valle yo creo que es de ese mismo talante, y más que nosotros, con los trabajos mal hechos de labranza como se llama, profunda, dañamos mucho la capa superficial [...]. (P7:PL.7:30)

Por ejemplo las lluvias de la semana pasada acá disminuyeron [se refiere a la producción de leche] como un litro en promedio..., por haber llovido, están en el barro, en el agua, granizo, ellas tienen que utilizar más energía en ese confort más no para producir leche, ellas quitan la producción de leche y utilizan más energía para regularse, y uno lo siente también en veranos, o sea al medio día el ganado toma mucha más agua, también se afecta la producción cuando hay calor, este ganado no está acostumbrado a temperaturas extremas. (P1:RG. 1:45)

Pues se enfermaron, hubo muertes también, aquí no se conocía eso, pero siempre, siendo..., pues teniendo un hato relativamente pequeño, aproximadamente unos 25, que se nos muera 1 o 2 animales pues es bastante, entonces eso sí tuvimos, muerte de animales [...]. (P4:LE.4:15) 
Estas afectaciones hacen que los productores tomen medidas para su mitigación o control del impacto que producen, a través de prácticas de cultivo que conducen a grandes gastos que contribuyen, en muchos casos, al incremento de las pérdidas económicas y a la quiebra de sus negocios. De acuerdo con el conocimiento de sus actividades ganaderas y experiencia expresan las medidas tomadas, como renovación de praderas, resiembra, movilización de animales, manejo de enfermedades y compra de comida, entre las más importantes, expresado de la siguiente manera:

Dos inundaciones en el mismo año, que eso fue gravísimo para todo el mundo, esto llevo a la quiebra. (P5:OT.5:12)

Perdí como 100 millones de pesos tranquilamente, en costos de oportunidad, todo lo que tenía sembrado se perdió, pues la finca sin producir nada, o sea todo, todo tocaba con comida para el ganado, bajas de producción, el arrendar una finca en otra parte, sí eso perdimos plata, pero.... (P2:HP.2:42)

[...] pero económicamente sí, porque lo que se produzca es compre comida, compre comida, eso es la parte más difícil, más, más difícil, porque todo lo que se produce es para comprar, sí, para comprar comida y pues he tratado últimamente pues como repartir, comida y arreglo de suelo, ya entrar como en esa disciplina. (P4:LE.4:37)

[...] la afectación de forrajes de las áreas de producción de forrajes pues que nos ha tocado traer comida de otros lados y, o mover animales también para otra finca, entonces pues eso nos ha traído un golpe económico grandísimo y bastante grande toda vez que fueron cuatro inundaciones en tres años seguidos, no nos estábamos recuperando de la primera cuando pum... la segunda, económicamente, físicamente en la pradera no nos estábamos todavía recuperando el 100\% y llegó la segunda inundación, y llegó el tercer año con dos inundaciones más, entonces pues hasta ahora el año pasado nos dio algo de tregua, [...].(P7:PL.7:37)

A mí lo que más me afecta es el volumen bajo [se refiere a cantidad de leche producida], volumen bajo por oferta de comida, básicamente es eso, la calidad pues le trabajamos para, le trabajamos mucho y tenemos muy buena calidad, pero a mí lo que más me afecta es el volumen. (P 4:LE.4:39)

Pues eso tiene un impacto económico gigantesco más que los TLCS más que cualquier otra cosa, pero pues yo creo que ahora nos podemos defender mejor, esperemos los nuevos retos a ver que sale. (P2: HP.2:15)

Los productores tienen su propia percepción de la vulnerabilidad y de riesgo en relación con su producción ganadera, ante los eventos de climáticos extremos y la expresan así: 
Claro que sí porque... ¿cómo produzco comida?, muy vulnerable para producir comida, quizá maneja más uno la situación de la sanidad animal, pero la producción de comida se nos torna muy complicada porque no podemos producir como debiera ser, entonces nos toca recurrir a comprar comida, entonces termina siendo..., se trabaja para comprar comida, sí, es básicamente eso. (P4:LE.4:20).

Hoy en día no saber qué va a pasar en el segundo trimestre del año o en el segundo semestre del año es gravísimo para cualquier persona que produzca en esta zona, porque no sabe para dónde va su negocio, para dónde va la rentabilidad de su negocio. Tú quisieras contar [...] con una producción estable, pero en un momento dado con cualquier fenómeno sobrenatural, llamémoslo así, nos vemos abocados a que de pronto vamos a reducir nuestros ingresos, de pronto el negocio en lugar de producir algo de utilidad de pronto va es a la quiebra. (P5:OT. 5:41).

Sí por eso, pero si no hay agua allá en la represa o que hagan como hacen en algunos municipios, prohibido el agua para uso agropecuario, ahí queda uno, y si llueve mucho pues la misma vaina, se vuelve a desbordar el río, o sea los eventos extremos ambos son potencialmente catastróficos, ambas cosas. (P2: HP.2:18)

[...] esta uno sujeto totalmente, es el único factor que uno no puede controlar, del resto puede uno controlar manejar los precios de los fertilizantes o los precios de los otros insumos, pero el clima si no lo puede uno, no tiene nada que hacer frente a eso. (P3:J Q.3:27)

La situación de vulnerabilidad que han vivido los productores del sector rural ha derivado en problemáticas de nivel social, como la tendencia migratoria, disminución en la mano de obra, cambios en el uso del suelo o cambios de producción. En cuanto a la tendencia migratoria y disminución de la mano de obra expresan:

Pues la gente de la región, a raíz de la escases de agua, el cultivo de la papa se vino a pique y frente también a los precios eso ha hecho que haya una migración grande, toda la juventud se está haciendo a las grandes ciudades, en la región sólo están quedando los viejitos por así decirlo, la productividad se ha bajado bastante. (P3 Jorge Quintero.3:34) [...] La mano de obra, por supuesto si los jóvenes se van no hay mano de obra. (P3 JQ.3:35)

[...] yo sé qué vecinos sí han tenido ese lío pues porque ellos sí quedaron bajo agua pues en la cuestión de invierno y pues personas muy allegadas muy cercanas aquí a la finca realmente les toco salir totalmente de la finca porque estaba totalmente inundada. (P7:P L.7:36)

No tienen para regar sus cultivos y eso crea que la gente se vaya, que haya migración del campo y eso me parece que no es deseable en ninguna parte porque no va a quedar quien trabaje. (P6: Ángela López.6:21) [...] nosotros estamos rodeados de mucho minifundio y se ha ido mucha gente para la ciudad porque han tenido muchas pérdidas económicas y se han tenido que ir porque no tienen de que vivir. (P6: ÁL.6:43) 
Teniendo en cuenta la importancia de que los productores estén organizados y que puedan estrechar lazos de solidaridad y apoyo que les permita la resiliencia, se explora sobre la capacidad que tienen de auto organizarse a partir de la necesidad de sobreponerse a eventos extremos:

[...] hemos hecho algo con los vecinos, y nos hemos integrado un poquito e inclusive en esas épocas duras de inundación pues lógico nos colaboramos en mover ganados, en permitir que los animales estén un tiempo en las fincas que no son tan inundables, mientras pasa por lo menos o baja la inundación, entonces eso ha acercado un poco más a los vecinos pero realmente al final se convierte en el esfuerzo de cada uno y cada uno se da sus soluciones no, esa parte gremial poco existe, de pronto la vivimos en un momento de crisis y de desastre ambiental pero realmente así como tener una..., como se llama, un gremio o algo que nos... no tanto un gremio algo que... nos..., no tanto un gremio sino algo que nos permita como ser solidarios desaparece con el tiempo la verdad, no la mantenemos. (P7: P L.7:44.116:116)

No existe red de apoyo, pero cuando el invierno es tan fuerte, que se dañan las vías, que todas esas cosas, sí la gente realmente colabora, ayudando con la mano de obra, pero son cosas porque nacen de dentro de la comunidad solitas, sin apoyo de nadie. (P6:Á L.6:56.152:152)

El papel que juega el Estado en el abordaje de soluciones a situaciones catastróficas derivadas de las variaciones de la variabilidad es fundamental para ellos, toda vez que requieren de políticas claras relacionadas con la planificación de la producción en el tiempo y en el espacio, que les permitan sobreponerse a estos eventos. Algunas de las percepciones que los productores tienen sobre el papel que ha tenido el Estado en su sector son:

Aquí ve que cada cual defiéndase como pueda, no hay políticas claras para todo el mundo, llámese con clima, llámese con medio ambiente, llámese con todo. Yo digo que falta mucha presencia de Estado y de ministerios y demás que verdaderamente pongan leyes claras y que ordenen tanto lo ambiental como lo hídrico y lo climático para ver hacia donde debemos guiarnos. (P5: OT.5:44).

Hay zonas especializadas en producción de leche, en producción de café, en producción de lo que sea, pero aquí no ha habido ministerio de agricultura que diga estos son los parámetros y hasta aquí vamos a producir el café, de aquí de esta zona en adelante vamos a producir leche, de esta zona en adelante vamos a sembrar papa, maíz, que sé yo, lo que sea los más ideal y lo más adecuado para cada región del país, pero jamás lo han hecho, aquí cada cual hacemos lo que queremos desafortunadamente porque no hay presencia, vuelvo y le digo, no hay presencia de Estado que diga, la norma va por aquí o estos es lo que se justa a estas necesidades de esta región, jamás lo han hecho. (P5: OT.5:45).

Prácticamente nulo, el Estado no llega con sus programas al productor se queda por el camino, la verdad es esa, el Estado salvo las capacitaciones de resto no se ha llegado con nada. (P3:JQ.3:42). 
No nunca, lo que nosotros hacemos lo hacemos de nosotros, pero del Estado presencia nada. (P6: ÁL.6:52).

Los productores a pesar de las diferentes adversidades que tienen en su día a día, se mantienen, luchan, buscan por todos los medios sobreponerse a la situación:

[...] tenemos la explotación hace más o menos unos 60 años está en el poder de nosotros, somos, mi hermano y yo somos los dueños actualmente y somos la tercera generación que tiene esta finca en nuestro poder. (P6:ÁL.6:2)

[...] yo realmente me he quedado en lo mismo aguantando pues, pues una estabilidad en la cuestión climática y en la producción para pues proseguir con esto que es algo más como cultural digo yo de seguir en lo mismo a pesar de los fracasos económicos que hemos tenido por tres años. (P7:PL.7:41)

Respecto de la posible corrupción que se presenta por parte de funcionarios del Estado en épocas de calamidad y desastre, expresan:

[...] a mí me han llamado a ofrecerme bonos, la gente recibe los bonos y a revenderlos y eso tampoco no me parece, no me parece, y eso fue muy común, creo que esa ayuda no ha llegado muy bien canalizada a los ganaderos, le han dado por allá a otras personas hasta arrendatarios que ya entregaron fincas y resultaron con sus semillas allá guardadas como un depósito y es la hora que le ofrecen a uno, mire tengo semilla, le vendo semilla, se la dejo más baratica. (P1:RG.1:28.117:117

[...] hay que llenar unos requisitos para poder acceder a eso porque no le venden a todo el mundo no, y tienen que controlarlo porque hay algunos que compran de eso para revender $y$ hacer el negocio. (P4:LE.4:47.168:168)

\section{Conclusiones}

A manera de conclusiones generales se presenta a continuación la ratificación de la condición de sujetos y actores sociales de los campesinos productores de leche en el Altiplano Cundiboyacense, lo mismo que una corta y nueva dimensión temática derivada de la interpretación de las percepciones expresadas por ellos en el escenario de estudio, que se presenta como resiliencia comunitaria.

\section{Los productores como protagonistas de su propio desarrollo}

Inicialmente hay necesidad de expresar que el reconocimiento de los productores de leche del Altiplano Cundiboyacense como sujetos o protagonistas del desarrollo de su propia vida, es decir, como personas capaces de 
tener proyectos, en este caso proyectos productivos relacionados con la producción de leche, con disponibilidad y disposición para elegir y juzgar de modo positivo o negativo sobre los eventos climáticos adversos, su incidencia, intensidad y frecuencia, y para formular posibles soluciones a la situación. Se ratifica por tanto su condición de personas válidas para ser consultadas no sólo sobre la problemática de la variabilidad climática sino, además, sobre las medidas necesarias o requeridas, según ellos, para enfrentar estos eventos tanto en épocas de lluvias como de verano.

Su capacidad de participación surge, como se puede apreciar por las respuestas, de su propia experiencia, de sus interacciones con los demás productores en la comunidad o el entorno social en el que se encuentran insertos y de su diario vivir, lo que les permite plantear sus propias opiniones, sensaciones, recuerdos, sentimientos, a partir de su percepción particular y propia fundamentada en su experiencia.

\section{Los productores como actores sociales del desarrollo de su comunidad}

Los productores muestran toda una disponibilidad de ánimo para abordar los inconvenientes derivados de los efectos de la variabilidad climática en sus sistemas productivos. Lo demuestran cuando expresan las múltiples actividades desarrolladas para mitigar los impactos derivados y las consecuencias de ellos: compra de comida para sus animales ante la imposibilidad de producirla por las inundaciones, grandes movimientos de tierra en busca de un mejor drenaje que evite las inundaciones, apoyo entre ellos en momentos de crisis, movimiento del ganado a otros predios en situación de menor riesgo, etc. Pero a lo único que expresan que no pueden enfrentar como quisieran son los cambios en la variabilidad climática que no pueden controlar, como las consecuencias e impactos que de ellos se originan.

Igualmente, la persistencia en la continuación de su actividad y en el afrontar los problemas derivados la asignan a su tradición, al hecho de que sus antepasados trabajaron estas tierras, al hecho de que varias de sus generaciones trabajaron y forjaron un futuro para ellos, el pertenecer a una familia que por años ha desarrollado las mismas actividades sin rendirse ni dar su brazo a torcer.

No obstante, no se configuran como actores o agentes del desarrollo de su comunidad, ante el hecho de que sus necesidades, sus opiniones sobre las actividades a desarrollar y su percepción de las razones por las que se presenta el fenómeno climático, no son tenidas en cuenta por quien le corresponde tomar las medidas o formular las políticas para abordar la mitigación y control de impactos y consecuencias. Entonces, si a la luz de las tesis de Touraine, ante el hecho de carecer de "conciencia ciudadana" que los configura como actores sociales, ¿̇cómo explicar el rechazo a la "adaptación" con la que desde la ley 1523 de 2012 se identifica la resiliencia? A continuación, se responde este interrogante. 


\section{Resiliencia comunitaria como categoría emergente}

Dado que esta es sólo una reflexión sobre los hallazgos que hasta el momento se han encontrado en la investigación en proceso de ejecución, se alude, a manera de conclusión, a una posible categoría que parece emerger, la cual se define como la "resiliencia comunitaria", entendida como "la capacidad de los seres humanos de superar los efectos de una adversidad a la que están sometidos e, incluso, de salir fortalecidos de la situación" (Melillo, 2006a, p. 64). Es una definición válida tanto para la resiliencia individual como para la familiar, grupal y comunitaria, pues se tienen en cuenta los factores de riesgo que acechan a cada persona en particular, a una familia o a una comunidad entera, como es el caso de los productores de leche del contexto sociocultural del estudio.

Esta es una categoría que difiere de la expuesto desde la ley colombiana y los organismos del Estado responsables de la política ambiental y climática en Colombia, pues se aparta de la "capacidad de adaptación" con la que se identifica "resiliencia" en la ley 1523 de 2012, adaptación que se entiende como "el ajuste de los sistemas naturales o humanos a los estímulos climáticos actuales o esperados o a sus efectos, con el fin de moderar perjuicios o explotar oportunidades beneficiosas", tal como lo anuncia su numeral 1 del artículo 4 . Estas afirmaciones de la Ley desconocen al productor de leche como actor social o agente del desarrollo de la comunidad rural en la que se encuentra inserto, no lo visibiliza como agente que participa de la construcción de su sociedad y puede incidir en sus cambios, pues la adaptación formulada, en este caso, se vuelve un mero intento individual de sobrevivir sin mayor esfuerzo por afrontar las condiciones adversas derivadas, es una adaptación positiva a la adversidad o, lo que es lo mismo, una mera conformidad social, en este caso, una adaptación positiva a la variabilidad climática o una conformación silente con la situación catastrófica presentada.

La resiliencia comunitaria por el contrario, se fundamenta más en la epidemiología social que en los enfoques clásicos referidos a casos particulares y, en este sentido, describe los pilares de la resiliencia comunitaria (Melillo, 2006b, p. 80), los que son perfectamente congruentes con las percepciones de los productores en el escenario de estudio. En efecto, estos pilares son:

a) Autoestima colectiva. Esta involucra la satisfacción por la pertenencia a la propia comunidad y se evidencia cuando los productores afirman "somos los dueños actualmente y somos la tercera generación que tiene esta finca en nuestro poder" (P6: ÁL.6:2), es una satisfacción que trasciende lo que se siente por pertenecer a una familia para satisfacerse de pertenecer a una comunidad en donde esa familia se reconoce, quiere y es respetada.

b) Identidad cultural. La constituye el proceso interactivo que a lo largo del desarrollo implica la incorporación de costumbres, valores, etc., que se transforman en componentes, proporcionando la sensación de 
pertenencia. Es esa misma pertenencia que los hace afronta la adversidad sin desistir de hacerlo, es lo que les hace expresar que “...pues proseguir con esto que es algo más como cultural digo yo de seguir en lo mismo a pesar de los fracasos económicos que hemos tenido por tres años”. (P7: PL.7:41).

c) Honestidad estatal. Es como una contrapartida de la corrupción que desgasta los vínculos sociales. Puede que en sentido estricto no exista corrupción estatal, pero lo que no existe es la presencia del Estado.

d) Solidaridad entre integrantes de la comunidad. Se presenta como fruto de sus lazos afectivos implícitos en la sensación de pertenencia a un colectivo humano. Se evidencia cuando los productores afirman: "cuando el invierno es tan fuerte, que se dañan las vías, que todas esas cosas, sí la gente realmente colabora, ayudando con la mano de obra, pero son cosas porque nacen de dentro de la comunidad solitas, sin apoyo de nadie". (P6: AL.6:56.152:152)

e) Humor social. No obstante las catástrofes que se afrontan por el cambio en la variabilidad climática y los impactos y consecuencias que de ella se derivan, los productores siempre tienen una atención y una sonrisa que brindar, no presentan socialmente hablando resquemores ni mal genio derivados de la situación.

Además, existe un sexto pilar de la resiliencia comunitaria, posiblemente el más importante de todos los descritos hasta ahora: la capacidad de pensamiento crítico. Esta se define como la voluntad de extender el deseo personal de bienestar a todos los otros y la capacidad de comprometerse con valores (Melillo, 2006b, p. 81). Es aquel que indaga, analiza más allá del consenso público primario y no acepta el statu quo social como un destino inexorable. Los productores sujetos de estudio manifiestan percepciones críticas sobre el papel del Estado para abocar las garantías requeridas para soluciones derivadas de las crisis climáticas, cuando expresan: "falta mucha presencia de Estado y de ministerios y demás que verdaderamente pongan leyes claras y que ordenen tanto lo ambiental como lo hídrico y lo climático para ver hacia donde debemos guiarnos" (P5: OT.5:44); "El Estado no llega con sus programas al productor se queda por el camino, la verdad es esa, el Estado salvo las capacitaciones de resto no se ha llegado con nada. (P3: JQ.3:42); “Lo que nosotros hacemos lo hacemos nosotros, pero del Estado presencia nada” (P6: Á L.6:52).

Esta capacidad de pensamiento crítico es un pilar fundamental de la resiliencia comunitaria, permitiendo calificar de resiliente la conducta de los productores de leche quienes han enfrentado pruebas, superándolas hasta el momento. Esta resiliencia es producto de la capacidad de afirmarse en un pensamiento crítico, es decir, de no adaptarse socialmente, como expresa la Ley colombiana, al silencio, a la resignación, es una especie de respuesta a la adversidad, que los impulsa a expresar a viva voz su descontento con el papel del Estado; eso los hace actores o agentes del desarrollo de la sociedad. 


\section{Referencias Bibliográficas}

BUSSO, G. (2001). Vulnerabilidad social: nociones e implicancias de políticas para Latinoamérica a inicios del siglo XXI. En: Seminario internacional las diferentes expresiones de la vulnerabilidad social en América Latina y el Caribe, Santiago de Chile: s.n.

CONGRESO DE LA REPÚBLICA DE COLOMBIA. (2012). Ley 1523 del 24 de abril de 2012. [En línea] Available at:

http://www.alcaldiabogota.gov.co/sisjur/normas/Norma1.jsp?i=47141

[Último acceso: 86 2014].

CORPOICA. (2004). Caracterización de los sistemas de producción de leche del trópico de altura de los departamentos de Boyacá y Cundinamarca, Mosquera : s.n.

---. (2012a). Zonificación del impacto de la oscilación ENOS sobre las condiciones climáticas del Altiplano Cundiboyacense como base para orientar la generación de transferencia de tecnología agropecuaria y reducir la vulnerabilidad climática. Informe, s.l.: s.n.

---.(2012b). Plan de manejo de los impactos en el sector agropecuario ocasionados por la emergencia invernal., s.l.: s.n.

IDEAM. (2011). Metodología para el análisis de vulnerabilidad y análisis de riesgos asociados a la cuenca alta del Río Cauca. Programa de Integración de ecosistemas y adaptación al cambio climático en el Macizo Colombiano.. Bogotá : s.n.

IGAC. (2006) . Base cartográfica 1.100000. Limites municipales y departamentales. Bogotá : s.n.

MELILLO, A. (2006a). Realidad social, psicoanálisis y resiliencia.. En: Melillo, A.; Suárez E. \& Rodríguez, D. (comp,). Resiliencia y subjetividad. Los ciclos de la vida.. Buenos Aires: Paidós , pp. 63-76.

---. (2006b). Sobre la necesidad de especificar el nuevo pilar de la resiliencia. . En: En: Melillo, A.; Suárez E. \& Rodríguez, D. (Comp.) Resiliencia y subjetividad. Los ciclos de la vida.. Buenos Aires: Paidós, pp. 77-90.

TOURAINE, A. (1996). Juventud y democracia en Chile.. Revista Latinoamerica de Juventud, Issue 1, pp. 36-48.

---.. (2000). ¿'Podemos vivir juntos? La discusión pendiente: el destino del hombre en la aldea global.. Ciudad de México : Fondo de Cultura Económica. 